\title{
A review on magnetically assisted abrasive finishing and their critical process parameters
}

\author{
Chinu Kumari and Sanjay Kumar Chak* \\ Division of Manufacturing Processes and Automation Engineering, Netaji Subhas Institute of Technology, New Delhi 110078, \\ India
}

Received: 26 May 2018 / Accepted: 28 July 2018

\begin{abstract}
Magnetically assisted abrasive finishing (MAAF) processes are the precision material removal processes that have been applied to a large variety of materials from brittle to ductile and from magnetic to non magnetic. The MAAF process relies on a unique "smart fluid", known as Magnetorheological (MR) fluid. MR fluids are suspensions of micron sized magnetizable particles such as carbonyl iron, dispersed in a non-magnetic carrier medium like silicone oil, mineral oil or water. The MAAF processes overcome the limitation of abrasive flow machining by deterministically control the abrading forces by applying magnetic field around the workpiece. MAAF process is divided into two parts; one is magnetorheological finishing (MRF) and another is magnetorheological abrasive flow finishing (MRAFF). The MRAFF process gives better results as compared to results of MRF because it has additional reciprocating motion of MR fluid. In this article the attempt has been made to review various technical papers related to MRF and MRAFF. The experimental setups, process parameters, MR fluid, modeling \& optimization and applications are discussed in this paper. This review article will be useful to academicians, researchers and practitioners as it comprises significant knowledge pertaining to MAAF.
\end{abstract}

Keywords: magnetically assisted abrasive finishing (MAAF) / magnetorheological finishing / magnetorheological abrasive flow finishing / magnetorheological fluid / flexible brush

\section{Introduction}

Material removal is the principal operation carried out by a majority of manufacturing industries. Every product we use in day to day life has used machining process either directly or indirectly. In the past three decade, researchers were always striving to enhance the surface roughness up to nanometric level $[1,2]$. Surface finish is achieved traditionally by Grinding [3], Lapping [4] and Honing [5] by the use of abrasives. The main limitation of traditional processes: (i) shape and size of workpiece is fixed in grinding. There is no flexibility of choosing the workpieces other than cylindrical and plane. (ii) Honing is only for internal finishing [6]. (iii) Lapping is very slow process [7]. Low machinability with a new material is the main problem associated with these traditional machining processes. Moreover, the above processes have no control on finishing process. The problems associated with above mentioned methods for surface finishing has been successfully overcome by advanced nanofinishing techniques during the last decade. Comparison of surface finish obtainable by different finishing processes is given in Table 1 [7].

\footnotetext{
* e-mail: sanjaykchak@yahoo.com
}

Nano-finishing is the most important area of micro manufacturing and it is widely used for fabrication of highly precise and accurate components. Surface roughness of the finished surface is important because it carries the functional properties such as wear resistance and power loss due to friction [8]. The ultimate precision is achieved when the chip size approaches sub-nanometer size $\sim 0.3 \mathrm{~nm}[9]$. In the nanometer range material is removed in the form of atoms or molecules individually or in groups. Advanced processes are classified according to removal of atoms and molecules from there surface. Some processes such as elastic emission machining [10] and ion beam machining [11] work on the principal of removing atoms and molecules directly from the surface. Other processes based on abrasive wear remove them in cluster. Abrasive flow machining (AFM) is also one of the advanced abrasive based finishing. But the main limitation of AFM process is incapability to control the finish surface value. Deterministic control over in-process finishing forces is an important consideration for Nano-finishing with close tolerances and without damaging surface topography. The major bottleneck in existing finishing technologies lies in the incapability controlling the final surface finish by deterministically controlling abrading forces. The nature and strength of bonding material used to hold the abrasive 
Table 1. Surface finish achievable by different finishing processes [7].

\begin{tabular}{llll}
\hline Sr. No. & Finishing process & Workpiece & Ra value $(\mathrm{nm})$ \\
\hline 1 & Grinding & - & $25-6250$ \\
2 & Honing & - & $25-1500$ \\
3 & Lapping & - & $13-750$ \\
4 & Abrasive flow machining (AFM) WITH SiC abrasives & Hardened steel & 50 \\
5 & Magnetic abrasive finishing (MAF) & $\mathrm{Stainless} \mathrm{steel}$ & 7.6 \\
6 & Magnetic Float Polishing (MFP) with $\mathrm{CeO}_{2}$ & $\mathrm{Si}_{3} \mathrm{~N}_{4}$ & 4.0 \\
7 & Magnetorheological Finishing (MRF) with $\mathrm{CeO}_{2}$ & Flat BK7 Glass & 0.8 \\
8 & Elastic Emission Machining (EEM) with $\mathrm{ZrO}_{2}$ abrasives & Silicon & $<0.5$ \\
9 & Ion Beam Machining (IBM) & Cemented carbide & 0.1 \\
\hline
\end{tabular}

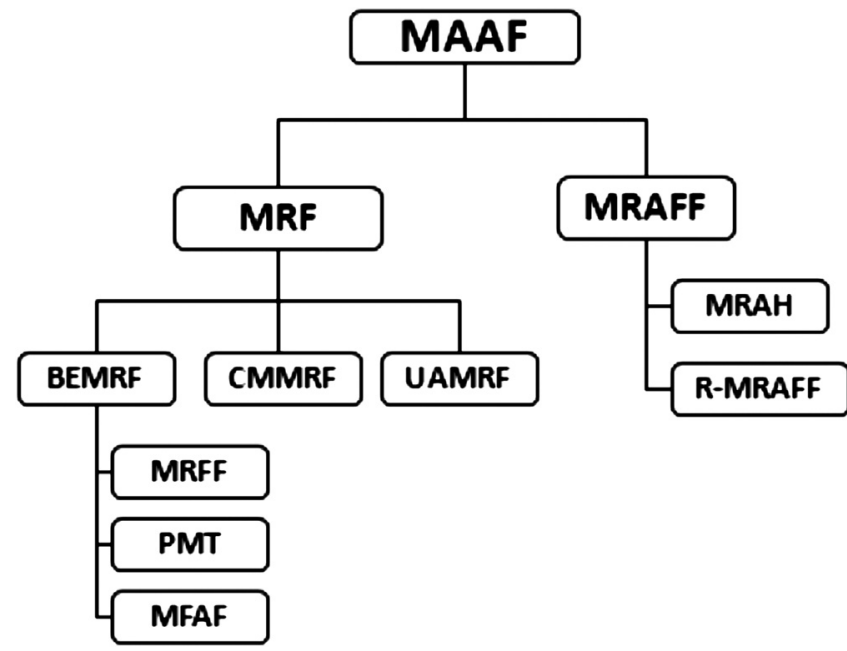

Fig. 1. MAAF, its types and their variants.

particles together determine the extent of abrasion and quality of the finished surface. Advanced fine finishing processes in which efforts were made to precisely control the abrading forces are magnetic abrasive finishing (MAF) [12], magnetorheological abrasive honing (MRAH) [13], magnetorheological finishing (MRF), magnetorheological jet finishing (MRJF) [14], magnetorheological abrasive flow finishing (MRAFF) [15]. In MAF, MFP, MRAFF and MRJF, the magnetic field is used to control the abrading forces.

Surface roughness and material removal rate are the responses mainly measured in these magnetically assisted abrasive finishing (MAAF) processes [16-18]. These responses depend on the operating conditions, Magnetorheological (MR) fluid, workpiece geometry and type of electromagnet used. These MAAF process are the new advanced techniques. In the present article, MAAF is classified into two types of finishing processes; MRF and MRAFF as shown in Figure 1. These processes are explained below with their variants.

Due to paucity of literature available regarding MAAF process, continuously work has been progressed regarding this process. Researchers have developed a variety of experimental setup for MRAFF process according to the complexity of the job. Today the demand of society is nanofinishing of the freeform surfaces owing their use in the aerospace sector, moulds and dies, medical implants, automotive components, etc.

The present article attempts to report various experimental setup of MRF and MRAFF. Process parameters of MAAF processes have been studied and their comparison among them was also done. This review is based on the reported experimental works and future research issues.

\section{MRF process}

Magnetorheological finishing (MRF) is the nano surface finishing technology. Mostly the optical surfaces are polished in a MR finishing slurry [19-23]. Researchers developed several MR fluids, each consisting of micron size magnetic carbonyl iron particles (CIP) and nonmagnetic polishing abrasives suspended in a carrier liquid [24-28]. Solid's concentration in excess of $42 \mathrm{vol} \%$ makes these MR fluids highly viscous. Carbonyl iron (CI) is important for the stiffness it brings to the MR fluid in the magnetic field, the polishing abrasive is necessary for high removal rates and smoothing of the part surface, and the carrier liquid is critical for establishing the correct polishing chemistry and the rheology necessary for fluid stability. Optical surfaces are usually made of brittle materials such as glass which tends to crack while it is machined with conventional process like grinding, honing and lapping [29-36]. The main advantages of the MRF process is that it carries away the heat and debris away from the polishing zone, does not load up as in grinding wheel and it is flexible and adapts the shape of the part of the workpiece which is in its contact [37]. In MRF process, the MR fluid shape and stiffness can be magnetically manipulated and can be controlled by magnetic flux density [38]. The mechanism involved in MRF of material removal and surface finish is basically comprises three different modes as shown in Figure 2.

- The abrasive particle held by chains of iron particles;

- the bunch of iron and abrasive particles move in the forward direction and shears/removes a very small amount of material in the form of micro-chip;

- when this bunch of iron and abrasive particles moves further, it separates the micro-/nano-chip from the work piece. 


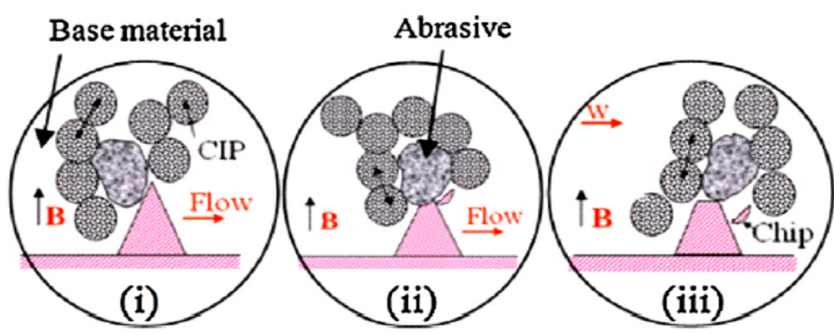

Fig. 2. Mechanism of material removal in MRF [38].

For the optical surfaces like lens center for optics manufacturing, in Rochester, N.Y. has developed a technology to automate the lens finishing process known as MRF [39]. Figure 3 shows that a convex, flat or concave workpiece can be positioned above a reference surface in the MRF process.

A MR fluid ribbon is deposited on the rotating wheel rim. Transient work zone or finishing spot is created by stiffened region by applying magnetic field [40]. When the lens rotates on a spindle at a constant speed about its radius of curvature through the stiffened finishing zone there is surface smoothing, removal of subsurface damage and figure correction are accomplished [41,42]. Converging gap between the part and carrier surface created a shear stress which is mainly responsible for material removal. The zone of contact is restricted to a spot, which conform perfectly to the local topography of the part [43].

\subsection{Variants in MRF}

The MRF is a MR fluid based finishing process so that most of their parameters are fluid based. The variants of MRF have been developed for betterment in the process to get improved results [44-52]. The researchers have done various experiments in terms of types of magnet used, shape of flexible ball created at the tip and combination of MRF with other conventional machining processes like chemical and ultrasonic machining. There are two ways to classify the variants of MRF machining process:

- processes in which all constituent processes are directly involved in the material removal and surface finish.

- the processes which only assist in removal/finishing by changing the condition of machining in a positive direction.

The MAAF processes can be classified into two parts; one is MRF process and another one is MRAFF process. In MRF, earlier magnetorheological slurry is used to finish the surface topography. But this process can finish only the concave, convex and flat surfaces due to restriction on relative movement of finishing medium and workpiece. The MRF is incapable to finish 3D intricate shaped surfaces. To overcome the above mentioned limitation a new precision nanofinishing process of 3D surfaces using ball end MR finishing (BEMRF) tool was developed. Thus, BEMRF process is the one of the variants of MRF process. The other process called MRFF was also developed with use of different type of MR fluid. However, the process mechanism remained almost the same as BEMRF process. In other variant of MRF named MFAF process for concen-

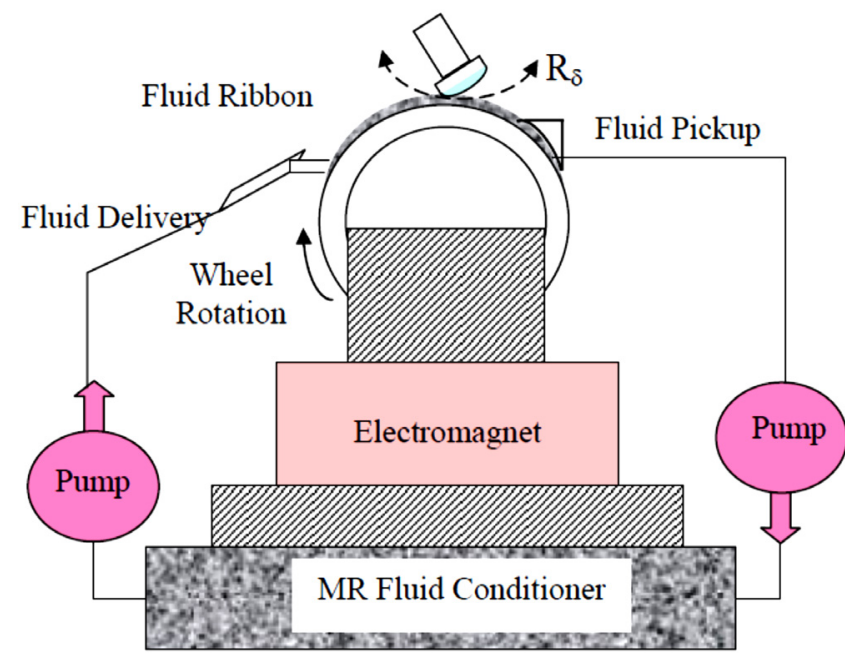

Fig. 3. Vertical MRF machine set-up [39].

trating the magnetic lines at the center of workpiece magnetic fixture is developed. Two types of magnets: permanent and electromagnet were used by the researchers [53-57]. Heating of electromagnet coil reduces the viscosity of MR fluid which can be avoided by using permanent magnet in place of electromagnet. The MRF process was hybridized by using chemical machining and ultrasonic machining to improve the finishing [58-61]. Variety of materials can be finished by MRF from hardness varies from 140-160 VHM. Both magnetic and non magnetic material can be finished upto roughness value $8 \mathrm{~nm}$.

Table 2 shows the developments occurred with time in MRF process. To describe the process technology four elements are considered; machine, MR fluid, type of magnet used and type of workpiece used. In the 'Machine' element; parameters like total time taken by the process, working gap and feed rate is explained. The total time taken by the process depends on hardness of the process and type of process used during machining [62]. When the working gap is increased the magnetic flux density at the workpiece surface is decreased. Types of CIP used, their concentration and size, types of abrasive used and their concentration \& size are explained in the element called 'Medium'. The machine decides the extent of abrasion, the media decides the kind of abrasion and the magnet determines the magnetic flux used during the MRF processes. Type of workpiece also affects the response in MRF process. Material removal rate (MRR) and surface finish are the main response measured during the MRF process.

\subsection{Ball end magnetorheological finishing}

BEMRF is one of the variant of MRF in which ball end is created at the end of polishing tool. It overcomes the limitation of the earliest developed MRF process by finishing the intricate 3D surfaces with more finishing rate and surface accuracy. In BEMRF process the ball end shape of MR polishing fluid is generated at the tip of rotating tool which is used as a finishing spot. To stiffen this MR fluid and to create the flexible ball at the tip of the tool, 


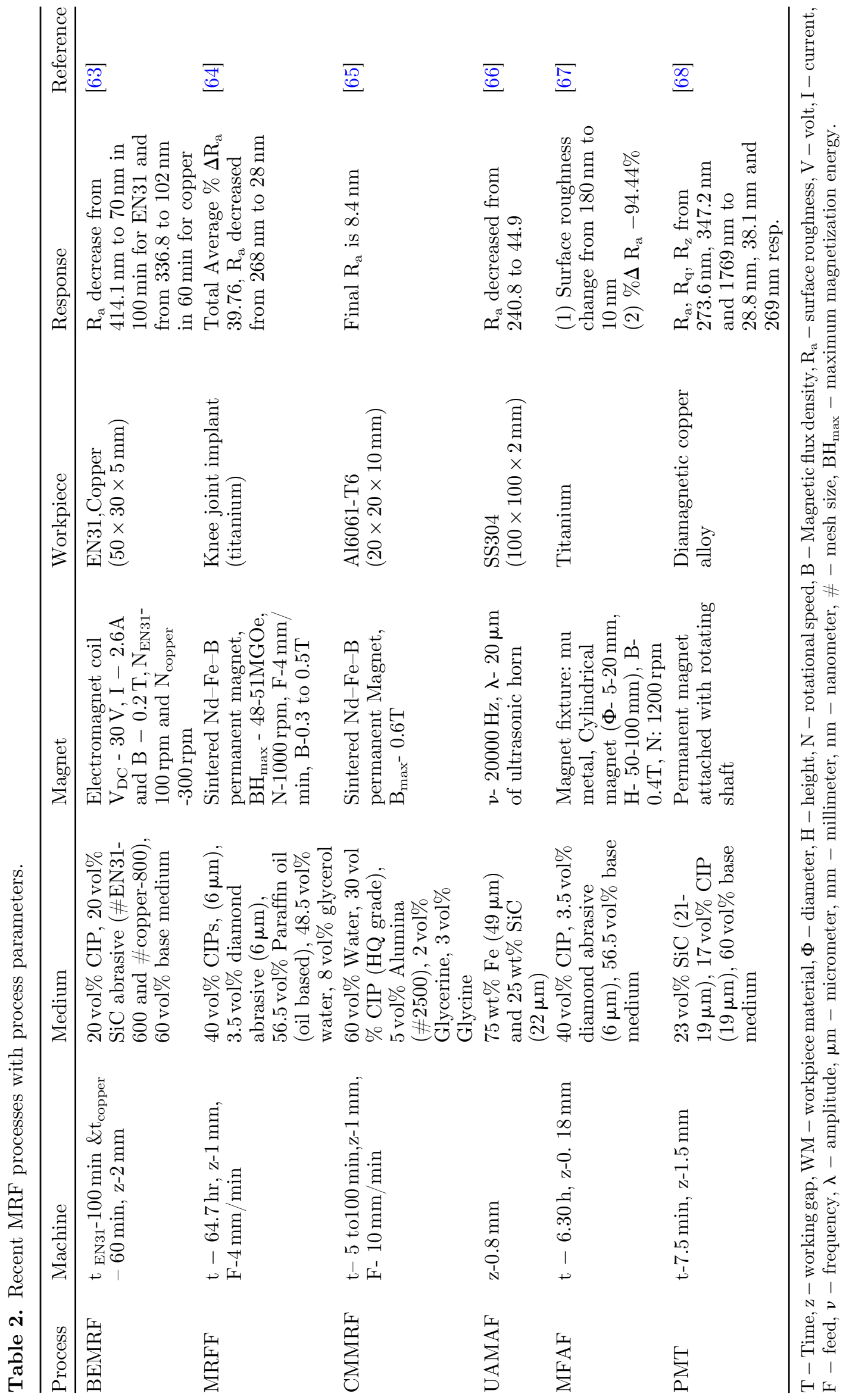




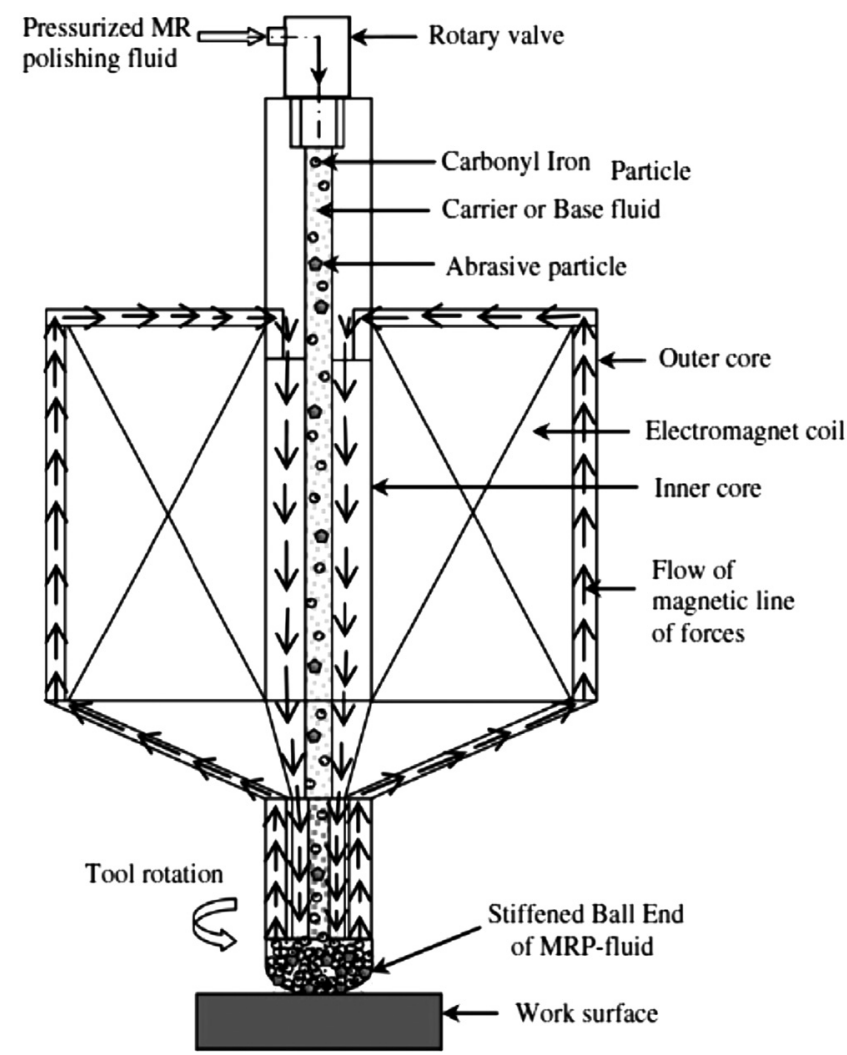

Fig. 4. Mechanism of formation of the stiffen ball end of MR polishing fluid at the tip of the tool [63].

the electromagnet coil was designed for this setup to obtain varying magnetic flux at the tip of tool. MR fluid was supplied through the core material of the electromagnet coil. The pressurized MR fluid enters axially from the upper end of the MR finishing tool. Once the magneto-rheological polishing (MRP) fluid reaches the tip of the tool, the magnetic CI particles of MRP fluid are aligned in the line and formed a chain like structure along with magnetic field direction available at the tip of the MR finishing tool as shown in Figure 4. The experiment was performed on two types of material, one is magnetic and another one is nonmagnetic. This method is superior for finishing the magnetic material as compared to nonmagnetic material because the magnetic lines of force are attracted towards the workpiece, form a very good shape of ball end of MR polishing fluid. But in case of nonmagnetic material these magnetic lines of force are diverted from inner core to outer core at the tip of the tool. More no. of cycles is required for finishing the nonmagnetic material. With this set-up, the roughness value decreased from $141.1 \mathrm{~nm}$ to $70 \mathrm{~nm}$ in finishing time of $100 \mathrm{~min}$ for ferromagnetic material. Results are shown in Figure 5.

The finishing of freeform surfaces is mostly done by CNC milling process and manually. Manual finishing processes are costly, tedious, inaccurate, labor intensive, and time consuming, and it relies mainly on the worker's skill. There is need to automate the finishing for freeform surfaces has become the most important issue to increase productivity and quality.

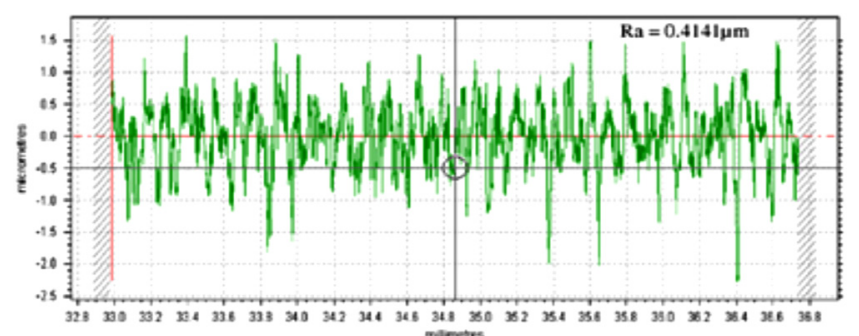

(a)

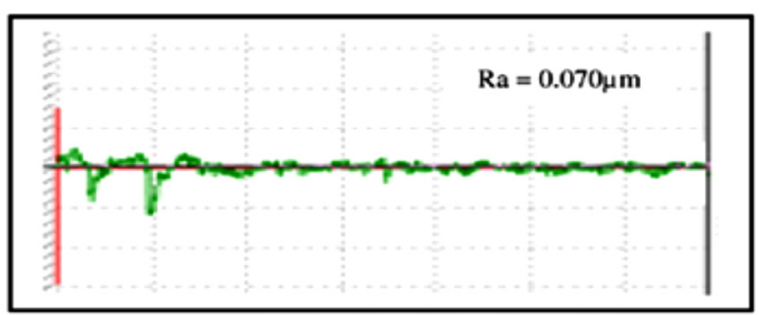

(b)

Fig. 5. SEM experimental images for EN8 workpiece before and after experiment [63].

Sidpara et al. [64], developed a magnetorheological fluid-based finishing tool for finishing the knee joint implant which has complex shape.

Different types of magnetorheological fluids are used to reduce finishing time. Three different kinds of MR fluids (oil based, water based and chemical) have been used to finish the knee joint implant. Firstly, the finishing of knee joint with oil based MR fluid was done. With this oil based MR fluid, the initial surface roughness value decreases faster owing to high initial sharp peaks that are comparatively easily removed with oil based MR fluid. After oil-based MR fluid, water-based MR fluid was used to further reduce the surface roughness. There is a little improvement in roughness value that is already finished with oil based MR fluid. There was a need to soften the surface by adding the chemical (HF and nitric acid) with water based MR fluid. It is observed that the surface roughness value decreases but the surface becomes dull owing to the chemical reaction between the MR fluid and workpiece. Hence, again water based MR fluid was used to improve surface reflection. Sidpara et al. [64] reported the best surface finish achieved through this process is of the order of $28 \mathrm{~nm}$. Most of MR processes make use of electromagnet for generating magnetic field. The coil of electromagnet at high current causes heating problem. Due to heating of coil, viscosity of MR fluid decreases. There is no provision for continuous use of electromagnet without chilling effect. This problem can be eliminated by use of permanent magnet [68]. It reduces the cost of apparatus and simplifies the setup. Two cylindrical magnets were selected for making the flexible brush at the tool end as shown in Figure 6. Those have been used to finish the diamagnetic copper alloy workpiece. Copper is diamagnetic in nature and difficult to finish at nano-level in shorter time. Here surface roughness from $273.6 \mathrm{~nm}$ to $28.8 \mathrm{~nm}$ in $7.5 \mathrm{~min}$ was achieved. To avail sufficient magnetic field in the finishing zone, Barman et al. [67], designed a magnet fixture to concentrate 


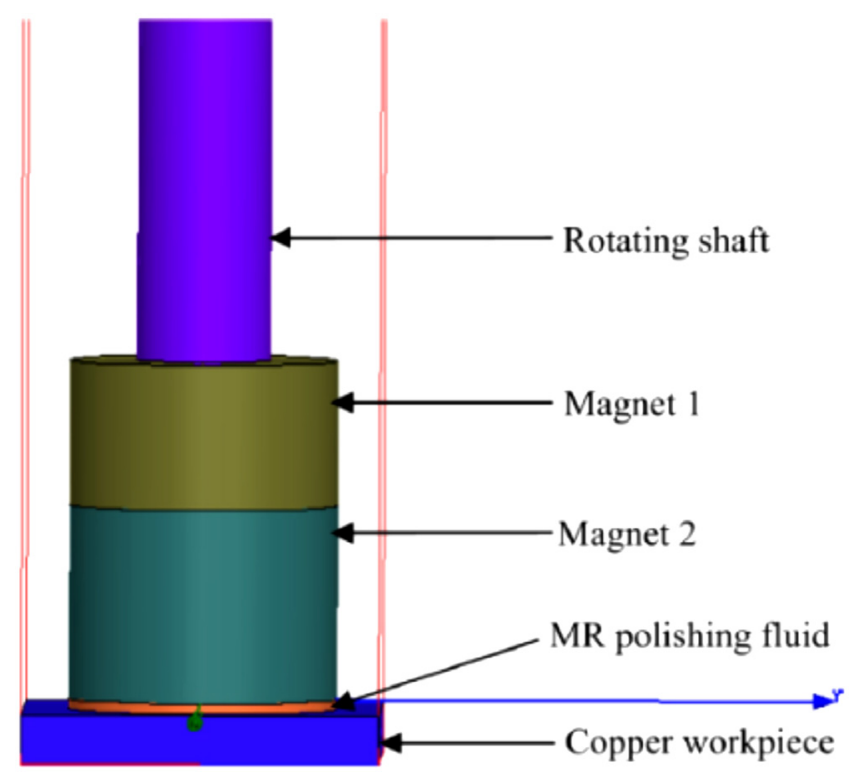

Fig. 6. Magnetic model of permanent magnets finishing tool along with MR polishing fluid and copper workpiece [68].

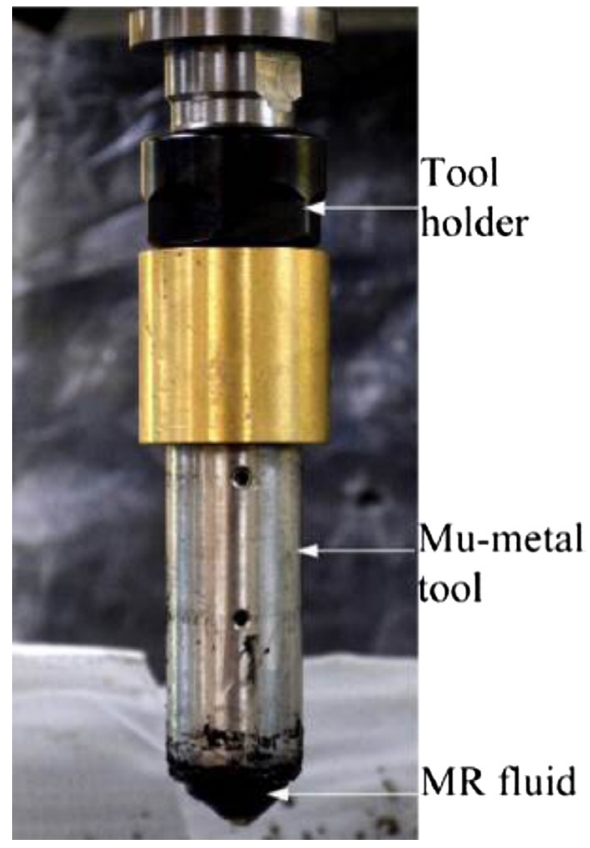

Fig. 7. Experimental set-up of MAFF process with Mu metal fixture [67].

all magnetic lines on the workpiece as shown in Figure 7. Mu metal is selected for magnet fixture due to its magnetic-field shielding property. The experimental results of MAFF process show that the optimum dimensions of permanent magnet are $15 \mathrm{~mm}$ diameter and $70 \mathrm{~mm}$ length.

\subsection{Chemical based MRF process}

To make the MRF process fast and to overcome the limitations of chemo-mechanical polishing (CMP) to finish the flat and cylindrical surface, the chemo-mechanical magneto-rheological finishing (CMMRF) [65] process was developed. It has combined essential aspect of both CMP [69] process and MRF process for surface finishing of silicon and copper. Chemical reactions are performed on the surface for making the soft layer on brittle materials e.g. Si, $\mathrm{SiO}_{2}, \mathrm{Al}_{2} \mathrm{O}_{3}$, etc. and formation of brittle layer on ductile materials [70] e.g. copper, aluminum etc. These chemical reactions protect the parent metal by making passive layer on the surface and alter the surface properties of the workpiece. Figure 8 shows the initial and final plot achieved during CMMRF process. Roughness value was reduced from $54 \mathrm{~nm}$ to $8 \mathrm{~nm}$.

\subsection{Ultrasonic based MRF process}

Various researchers performed experiments and found that the rate of material removal can be increased by giving vibration to the workpiece. The UAMRF is one of the hybridized process of MRF. It has advantages of both ultrasonic vibration and MRF. In ultrasonic assisted magnetic abrasive finishing (UAMAF) [66] process surface finish is achieved by a flexible magnetic abrasive brush [71] developed by ferromagnetic iron and non ferromagnetic abrasive particles (called magnetic abrasive powder or MAP) in presence of magnetic field and ultrasonic vibrations. Ultrasonic vibration increases the relative velocity of $\mathrm{MR}$ fluid, hitting the workpiece surface asperities. This results in improvement in the surface finish and material removal as compared to MRF process. The schematic diagram of UAMAF as shown in Figure 9 which comprises power source, piezoelectric transducer with a horn constituted a complete unit of an ultrasonic vibration generator setup which produce electrical signals. The transducer converts the electric signal upto $20 \mathrm{kHz}$ into mechanical vibration of workpiece. Researchers also developed a theoretical model of UAMAF process for normal force and horizontal force [72-75]. Normal force on the particles helps in indenting the particle on the work surface whereas horizontal force provides finishing torque that in-turn helps the particle to perform micro-machining.

\section{MR fluid}

MR fluids are the magnetic field-controllable fluids, their rheological behavior depends on the strength of the magnetic field. Normally, the rheological properties like viscosity and shear stress change with the physical properties of MR fluid such as chemical composition and temperature. In the "off" state of magnetic field, MR fluids appear similar to liquid paints and exhibit comparable levels of apparent viscosity ( 0.1 to $1 \mathrm{~Pa}$ s at low shear rates) [76]. Their apparent viscosity changes significantly $\left(10^{5}-\right.$ $10^{6}$ times) within a few milliseconds when the magnetic field is applied. Condition of MR fluid is shown in Figure 10 below with and without magnetic field.

The change in the viscosity is completely reversible when the magnetic field is removed. Once the magnetic field is applied, it induces a dipole in each of the magnetic particles. This dipolar interaction is responsible for the chain like formation of the particles in the direction of the 

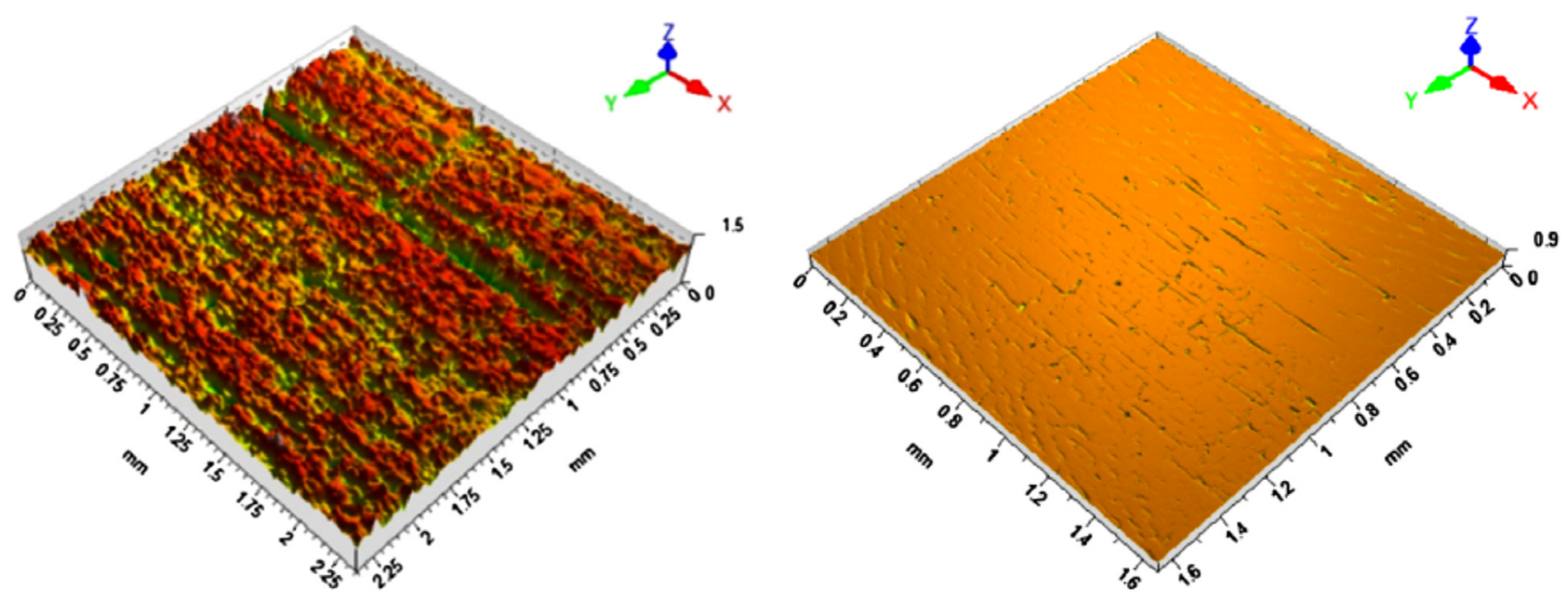

Fig. 8. Surface roughness value (a) before and (b) after CMMRF process [65].

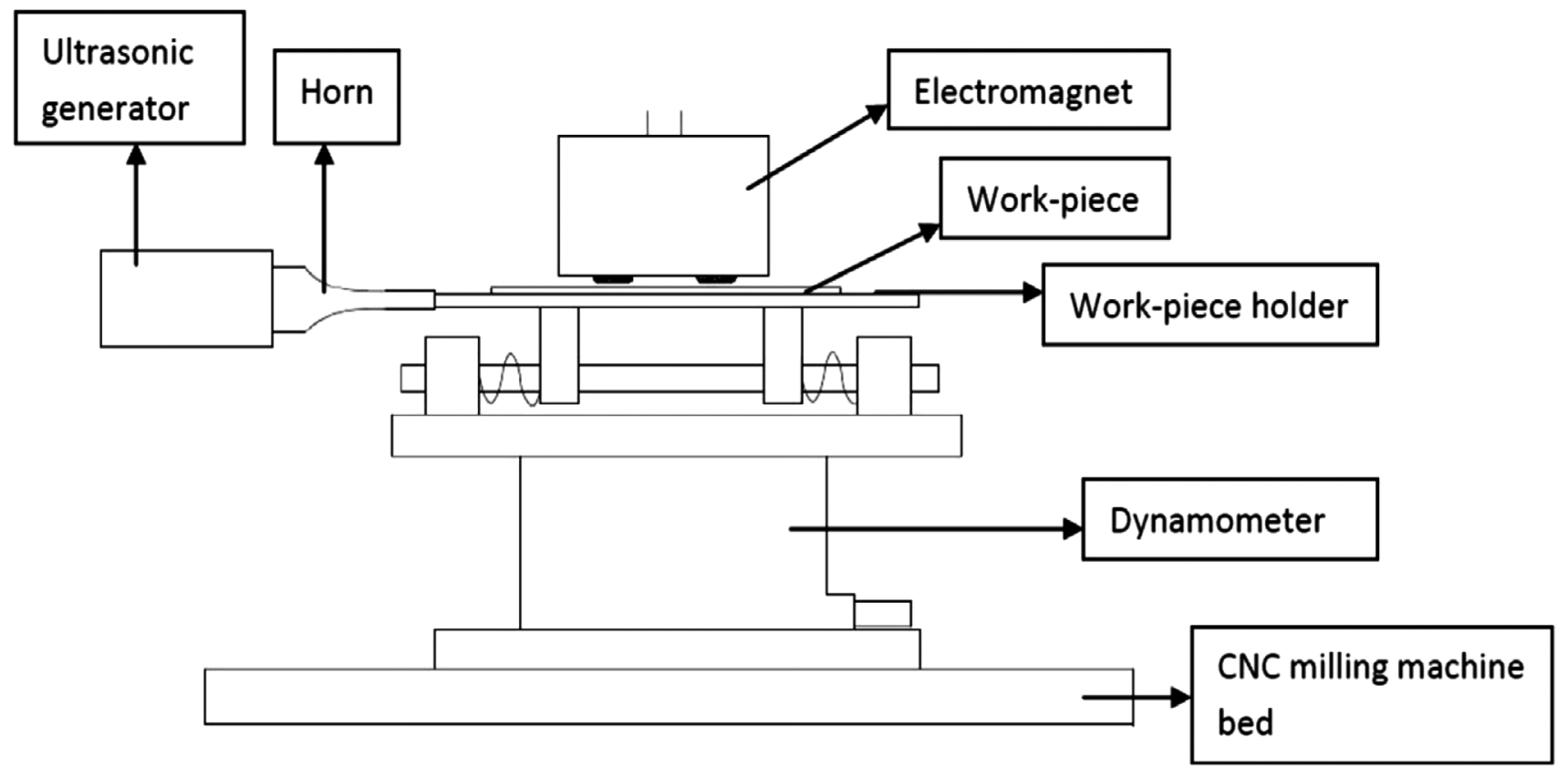

Fig. 9. Schematic of ultrasonic machining set-up [66].

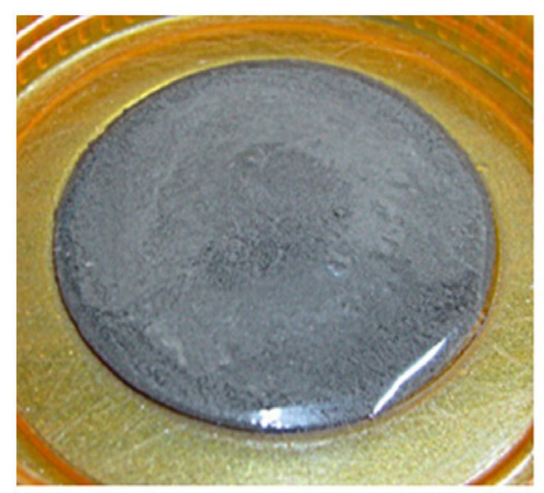

(a)

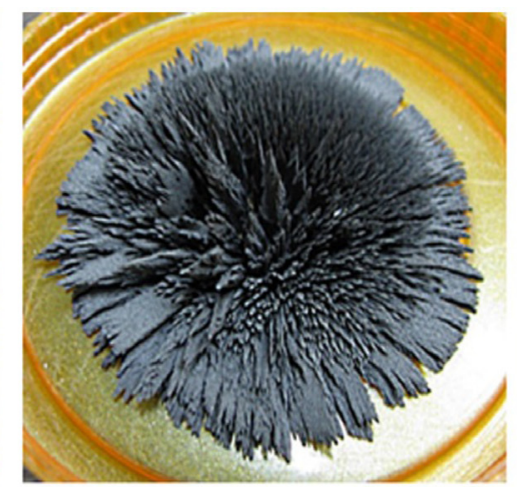

(b)

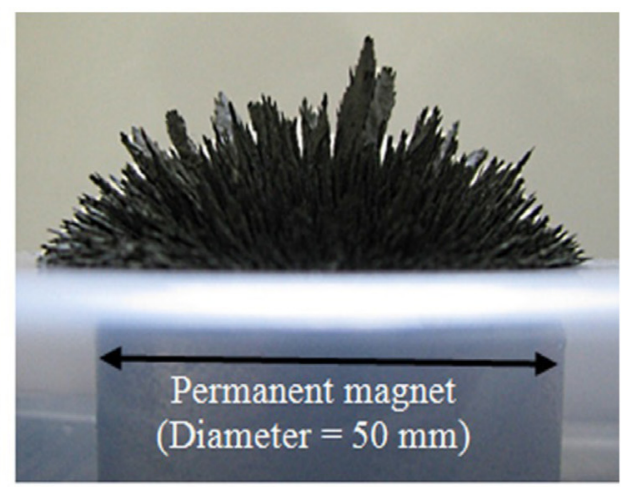

(c)

Fig. 10. Condition of MR fluid (a) without magnetic field (b) with magnetic field (c) side view [76]. 


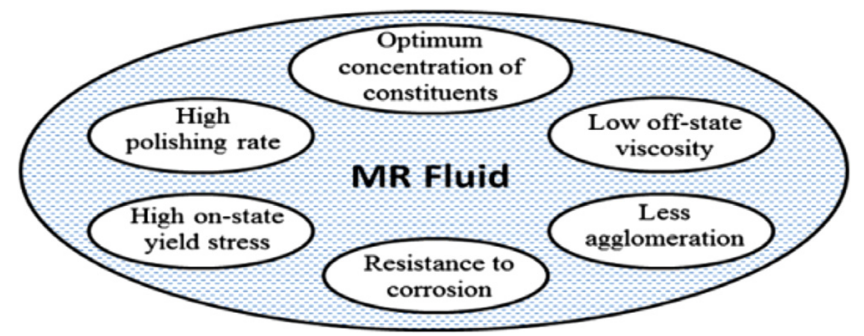

Fig. 11. Essential requirements of MR fluid [77].

magnetic field $[77,78]$. MR fluid is the prime concern for achieving desired output. Figure 11 shows the extensive requirements of MR fluid.

\subsection{Composition of MR fluid}

MR fluid consists of CIP, nonmagnetic abrasives and carrier fluids. These CIPs and abrasives are randomly oriented without the magnetic field as shown in Figure 12a. Under the effect of magnetic field, these particles align themselves along the lines of magnetic flux. These CIPs acquire dipole proportional to field strength and when dipole interaction exceeds their thermal energy these particles are aligned in the form of a chain as shown in Figure 12b. Energy is required to deform and rupture these chains. This microstructural transition is responsible for the onset of a large controllable yield stress as shown in Figure 12c. Their concentration should be kept optimum for getting best results. From the available literature, there are some examples of different concentration used by the researchers for experiments. For MRAFF process, used concentration was $20 \%$ CI powder (Grade CS from BASF, avg. particle size $18 \mu \mathrm{m}$ ), $20 \mathrm{vol} \%$ silicon carbide abrasive powder of $400 \mathrm{mesh}$ size and $60 \mathrm{vol} \%$ of viscoplastic base medium (20 wt.\% AP3 grease and $80 \mathrm{wt}$. $\%$ paraffin liquid heavy) was prepared [15]. The yield shear stress and viscosity of bi-disperse MRP fluid for all experiments were found maximum at $16 \mathrm{vol} \%$ CIPs of CS grade, $4 \mathrm{vol} \%$ CIPs of HS grade, $25 \mathrm{vol} \%$ abrasives and $55 \mathrm{vol}$ $\%$ base fluid [33]. The yield shear stress and viscosity of bidisperse MRP fluid were maximum as compare to monodisperse MRP fluid.

\subsection{Stability against sedimentation}

Researchers [25,27] have added submicron-sized particles (fumed silica FS) into CI based MR fluid. These spherical particles not only reduced sedimentation but also improved flocculation stability of the CI-based MR fluid without any noticeable change in the MR behavior.

\subsection{Additives}

Additives are suspending agents and anti-corrosion/antiwear components. Grease or other thixotropic additives are highly viscous fluid used to improve settling stability [26]. MR fluid contains a stabilizer such as glycerol to add viscosity to the MR fluid and to create conditions that help to keep the magnetic particles and abrasive particles in suspension.

\section{Magnetorheological abrasive flow finishing (MRAFF)}

In this chapter finishing methods like AFM and honing combined with MRF technology are explained and this newly developed hybrid process is termed as MRAFF [15] as shown in Figure 13. It takes advantages of both AFM and MRF.

This process has the capability of finishing complex internal and external geometries up to nano-level surface roughness value. It imparts better control on the process performance as compared to AFM process due to in-process control over abrading medium's rheological behavior through magnetic field. In MRAFF process, back and forth motion of magnetically stiffed MR fluid across the passage formed by workpiece and fixture is shown in Figure 14. MRAFF process and its variants with process parameters are explained in Table 3 below. MRAH and rotational-magnetorheological abrasive flow finishing (RMRAFF) are the two new processes which are discussed. The R-MRAFF is the one of the variant of MRAFF in which rotational motion is given to workpiece for more uniform and fast finishing the freeform surface. In other process named MRAH in which conventional honing is modify by magnetorheolgical effect of finishing fluid. The parameters come under machine are pressure during machining, time taken by the process, rotational speed of workpiece, reciprocating velocity of the MR fluid, working gap between electromagnet poles etc. The medium used during these processes is MR fluid and its concentration and size of abrasive particles and CIPs are used according to the requirement. The workpiece property either it is magnetic or nonmagnetic influence the response. Two output responses; material removal rate and surface finish are described here.

The knee joint in the past is finished by MAFF process. But this process has limitations in terms of larger finishing time and non-uniformity in the finished surface. To overcome these problems, Satish et al. [80], developed a new process known as R-MRAFF for finishing the complex surface with high finishing rate. With this process smooth mirror like surface finish is achieved by giving combined motion (axial and rotational) to the workpiece. For the complex surface, there are many inclination angles of the abrasives to indent on the workpiece surface. The final roughness value increases and finishing rate decreases with increase in the angle of inclination of surface. Because at higher angle of inclination the magnetic lines of forces are spread over the surface hence, flux density will get reduced. There is face-wise study of knee joint implant by the researchers for complete observation of the surface.

Large variation in the magnetic flux density is observed due to non-uniform gaps at different locations and different curvature on the surfaces. Because of non-uniformity in the freeform surface the surface near the magnet feel more magnetic field as compare to farthest surface. The average finishing rate in case of R-MRAFF is as high as 2 times as compared to the MAFF. The BEMRF process shows good results with magnetic materials hence a new process new process MRAH [79] is developed to finish any complex 


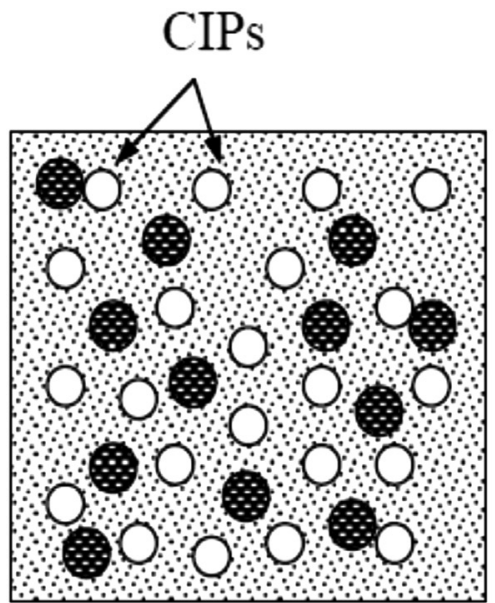

a

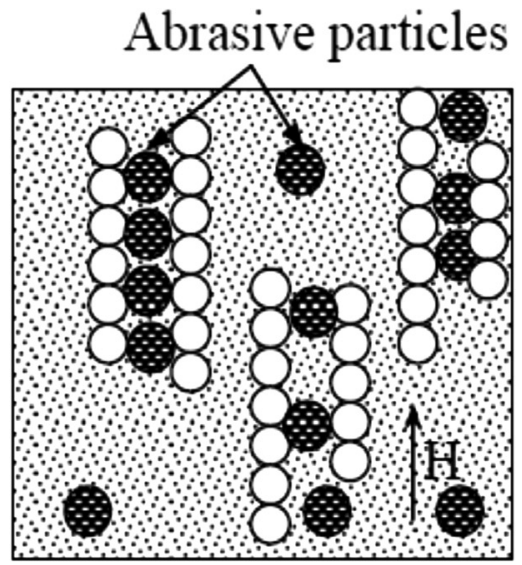

b

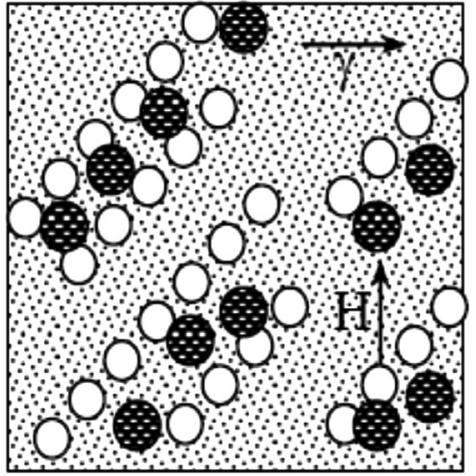

C

Fig. 12. Magnetorheological effect, (a) MRP-fluid at no magnetic field, (b) at magnetic field strength $\mathrm{H}$ and (c) at magnetic field $\mathrm{H}$ \& applied shear strain $\gamma$. [15].

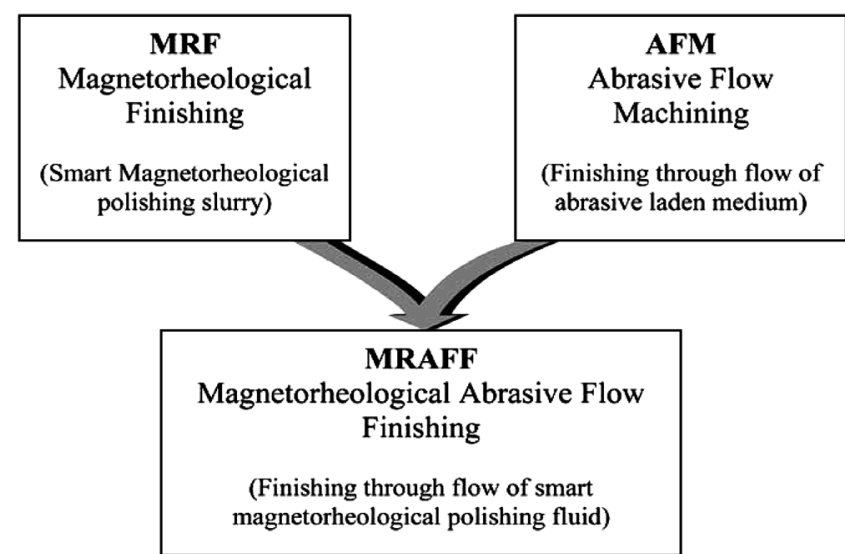

Fig. 13. Relation between the three process MRF, AFM and MRAFF [15].

shape non-magnetic workpiece with improved finishing rate. In MRAH, workpiece rotates and reciprocating motion is provided to the MR fluid. Schematic diagram of MRAH is shown in Figure 15 below. In MRAH [79] the workpiece rotates and reciprocating motion is provided to the MR fluid. Schematic diagram of MRAH is shown in Figure 14 below. When field density is increased the finishing fluid becomes stiff due to the formation of CIP chains. Due to this the roughness is decreased and material removal is increased. After a value of $0.6 \mathrm{~T}$, there is a meager improvement in roughness. There is no sufficient bonding between CIP and abrasive. The CIPs are attracted towards the pole faces of magnet after a threshold value. The experiment is performed on two types of material; one is magnetic and another one is nonmagnetic. There is only $6.7 \%$ improvement in roughness value for magnetic material because the CIP trapped all the abrasive near the workpiece. This phenomenon reduced the relative movement of abrasives on the workpiece surface. But for nonmagnetic, it shows better results and the improvement in roughness is $24 \%$.

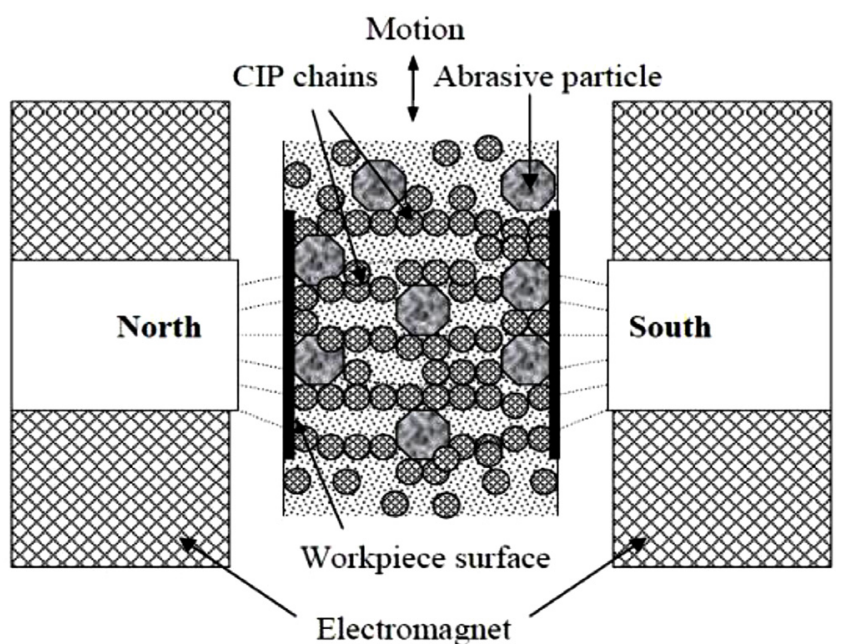

Fig. 14. Mechanism of magnetorheological abrasive flow finishing process [50].

\section{Modelling and optimization}

To understand the complexity of the process, it is necessary to construct a model either mathematical or theoretically to study the effect of various process parameters on the output responses; surface finish, MRR and finishing force. The model in general provides the information, which gives an insight into the nature of the phenomenon occurring in the real life situation. Through the MR fluid in MRF and MRAFF process, the normal and tangential forces are acting on the work pieces due to MR fluid pass through the magnetic field. Normal force is responsible for indenting the work piece surface and tangential force is responsible for removal of material from the work piece surface.

Ginder et al. [81] calculated the inter-particle forces and resulting shear stresses in magnetorheological fluid. The field due to a linear chain of particles in a fixed average magnetic induction is determined from finite element 


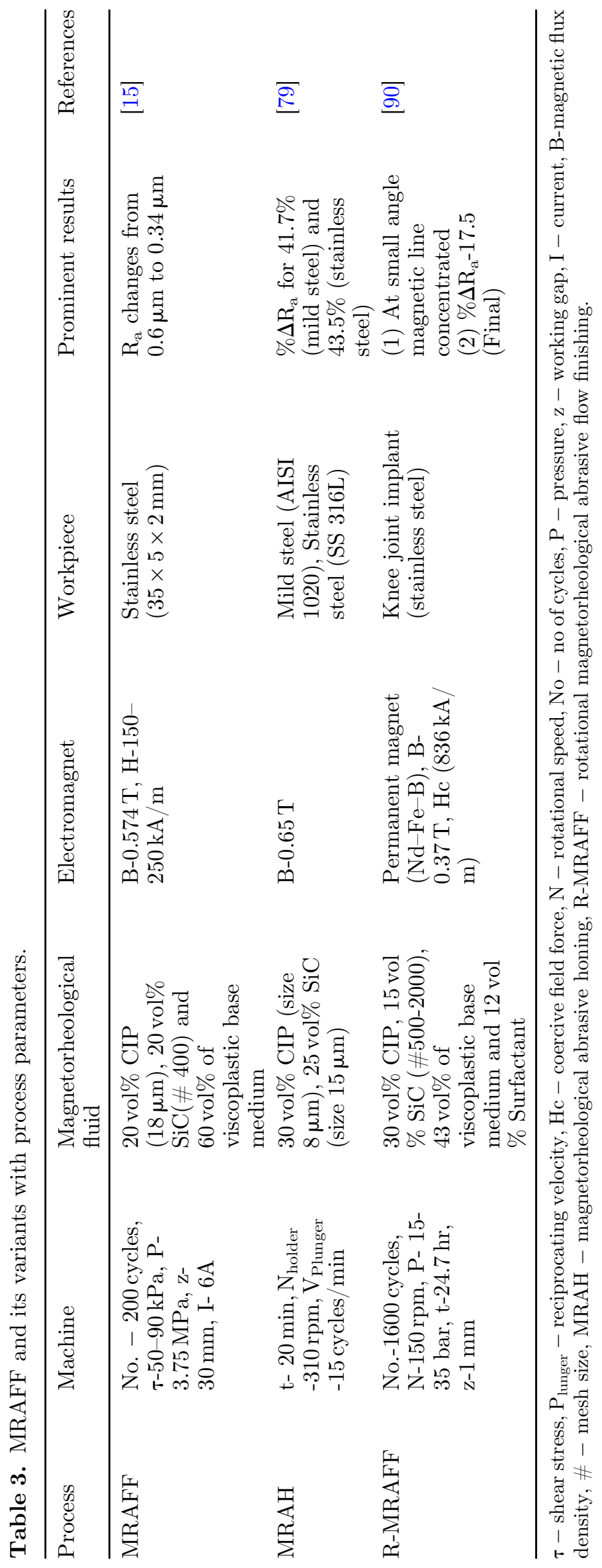


Table 4. Key capabilities of various MAAF process.

\begin{tabular}{lll}
\hline Process & Aim & Benefits \\
\hline BEMRF & $\begin{array}{l}\text { To create a flexible bush at the end of } \\
\text { magnetorheological polishing tool }\end{array}$ & $\begin{array}{l}\text { Any freeform surface can be finished by } \\
\text { maintaining proper gap }\end{array}$ \\
MRFF & $\begin{array}{l}\text { Use of different types of MR fluid like } \\
\text { chemical based, water based and oil based } \\
\text { Use of permanent magnet instead of } \\
\text { electromagnet }\end{array}$ & $\begin{array}{l}\text { Very hard material like titanium can be } \\
\text { finished upto nanometric level }\end{array}$ \\
MFAF & A magnetic fixture for permanent magnet & $\begin{array}{l}\text { Heating effect due to electromagnet coils can } \\
\text { be avoided and soft diamagnetic copper alloy } \\
\text { can be finished }\end{array}$ \\
MRAH & $\begin{array}{l}\text { It can be made possible to concentrate all } \\
\text { the magnetic lines on the workpiece }\end{array}$ \\
R-MRAFF & $\begin{array}{l}\text { with MR effect of MRF technology } \\
\text { wotation of permanent magnet fixture along } \\
\text { with reciprocating motion of MR fluid }\end{array}$ & $\begin{array}{l}\text { Finishing time can be reduced by rotating } \\
\text { the job with MR effect } \\
\text { High finishing rate and uniform finishing of } \\
\text { all faces }\end{array}$ \\
\hline
\end{tabular}

analysis. They concluded that the maximum shear strength of MR fluids varies as the square of the saturation magnetization $\mathrm{M}_{\mathrm{s}}$ of the particle. It means that the larger $\mathrm{M}_{\mathrm{s}}$ is possessed by the new particles in MR fluid which have higher yield strength.

Kordonski et al. [82], observed that the concentration of magnetic particles may change due to evaporation or leakage of carrier fluid, as well as particle sedimentation and the magnetic properties may change due to temperature, corrosion of particles or irreversible aggregation. They designed two types of sensors for in line measurements of magnetic particle's concentration in MR fluids; one is flow type sensor \& another is flash type sensor. The flow-type sensor is based on the principle of mutual inductance and is intended for measurements in pipes and channels. The flash-type sensor reacts to changes in the reluctance of the MR fluid and can be used for measurements in gaps, tanks, containers layer adjacent to the wall.

Yarmand [83] investigated numerically the thermal characteristics of turbulent Nano fluid flow in a rectangular pipe. CFD software (ANSYS-FLUENT) has been employed to simulate the results. From the variety of Nano fluids used by the author, the $\mathrm{SiO}_{2}$ generates the highest Nusselt number followed by $\mathrm{Al}_{2} \mathrm{O}_{3}, \mathrm{ZnO}, \mathrm{CuO}$ and the pure water. $\mathrm{SiO}_{2}$ has the least heat transfer coefficient but because of the lowest thermal conductivity it has highest Nusselt no. among the tested Nano fluids.

Sidpara et al. [84], designed a theoretical model of forces (normal and tangential) acting on the work piece to improve the in-depth understanding of the mechanism of material removal during MR fluid based finishing process. The normal forces are experienced by compression due to squeezing of MR fluid between the working gaps and tangential force due to rotation of carrier wheel. The magnets are used around the workpiece in these MAAF process. Due to magnetic field, magnetic levitation force acts on the MR fluid. In it, majority of abrasive particles move away from the magnet or towards the outer periphery of the ribbon and the workpiece surface [36]. Hence, material removal takes place by interaction of abrasive particles, which are gripped by stiffened MR fluid and workpiece. The levitation force $\left(\mathrm{F}_{\mathrm{m}}\right)$ is proportional to the gradient in the magnetic field and it is calculated by

$$
F_{m}=-V \mu_{0} M \nabla H
$$

where $\mathrm{V}$ is the volume of the non-magnetic body (abrasive particles), $M$ is the magnetization of the magnetic fluid, $\mu_{0}$ is the permeability of free space and $\nabla \mathrm{H}$ is the gradient of a magnetic field. Four parameters were selected for this study; volume concentration of CIPs, volume concentration of abrasive particles, working gap and wheel speed. They concluded that the normal and tangential force decrease with increase in working gap, however it will increase with increase in abrasive particles concentration but after a value of $3.5 \%$, forces starts to decrease because of more abrasives particles decrease the stiffness of MR fluid and break its chains with CIP. Firstly, the normal and tangential forces increase with increase in wheel speed but after the value of $300 \mathrm{rpm}$ the tangential force start decreasing due to flattening of the MR fluid ribbon. It is also observed that the theoretical normal force and tangential force model including squeeze force showed better agreement with experimental results as compared to the model without considering of squeeze force. Squeezing force is based on the theory of rolling. The concept is similar to the squeezing the metal in rolling as MR fluid in MRF.

Das et al. [85], designed and fabricated a capillary viscometer to study the effect of magnetic field on the rheological properties of the medium and finite difference method to evaluate the stresses developed during the process. Normal force on the abrasive particles is calculated from the applied magnetic field. it is concluded that the viscosity of the fluid increases in a third-order logarithmic function of magnetic field and also yield stress of the fluid increases with magnetic field as shown in the equation mentioned below

$$
\begin{aligned}
\mu_{p}= & 5918.57+10191.33 \ln (B)+6110.52(\ln (B))^{2} \\
& +1222.70(\ln (B))^{3} \\
\tau_{y}= & -33.10+67.09 \ln (B) / B+\frac{51.54}{B^{1.5}}
\end{aligned}
$$




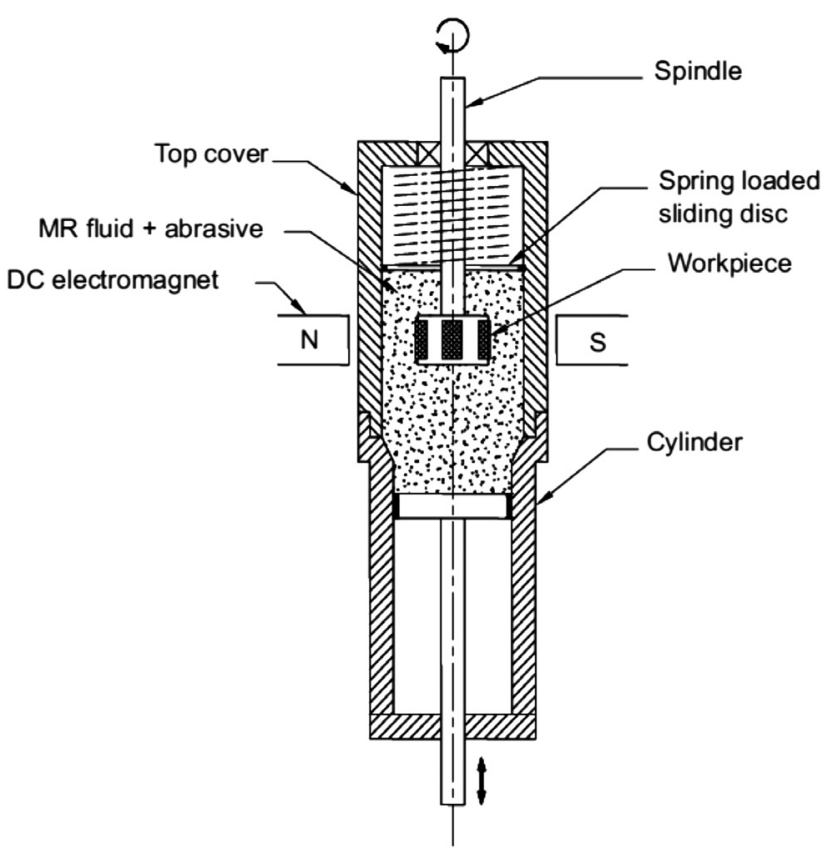

Fig. 15. Schematic diagram of the MRAH [79].

$\mu_{p}$ is the plastic viscosity (Pas), B is the density of magnetic field ( $\mathrm{T}$ ) and $\tau_{\mathrm{y}}$ is shear yield stress of the fluid $\left(\mathrm{N} / \mathrm{m}^{2}\right)$. At higher normal force the larger plug flow region of the flowing fluid is obtained with higher magnetic field due to the formation of strong structure of CIP chains with increased magnetic field. It is also concluded that the shearing takes place only when the shear force is more than the reaction of the workpiece. Singh et al. [86], studied the mechanism of material removal and magnetic field induced normal force during BEMRF process. It was explained that due to the induced normal force material removal and percentage change in roughness was increased. Also this magnetic field induced normal force decreased with increase in working gap. For more improvement in BEMRF process [87], it was required to plan a theoretical model for this process. In this model, theoretical understanding of surface roughness and mechanism of material removal associated with ferromagnetic workpiece is involved. There are two forces acting; one is magnetically induced normal force and another one is shear forces acting on the abrasive. This normal force is mainly responsible for penetration of abrasive into the workpiece surface and shear force model aids in the understanding of three body wear mechanism between the abrasive and the workpiece surface as shown in Figure 16.

\section{Applications of magnetic assisted abrasive flow machining}

Various researchers have used magnetic assisted abrasive finishing (MAAF) technique for finishing various components used in electronics, optical and biomedical fields to the required level of accuracy as explained in Table 3 . The various applications of MAAF process are discussed below.

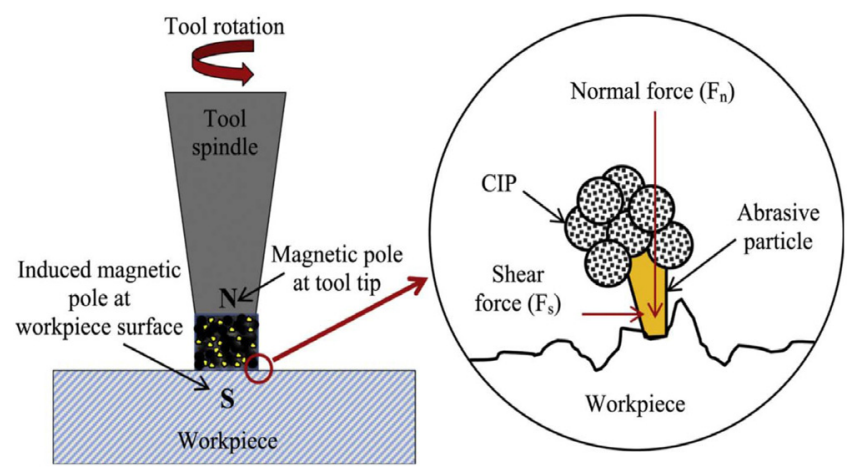

Fig. 16. Forces associated with BEMRF process [87].

\subsection{Machining of electronics and industrial components}

Silicon crystal with nano-level surface finish has many applications. It is used as IC chip in semiconductor, silicon strip mirror for cryogenic applications and synchrotron beam line and substrate material for X-ray mirrors. single point diamond turning is commonly used to finish silicon crystal but this process imparts turning marks on the final finished silicon surface. Therefore, MRF is more suitable for silicon finishing as compared to other processes.

Sidpara et al. [35] attained a final roughness value of silicon crystal as low as $8 \mathrm{~nm}$ from the initial value of $1300 \mathrm{~nm}$. Yin et al. [36] developed abrasive assisted technology to polish micro bores in metal- 400 and $500 \mu \mathrm{m}$ bores in steel $\mathrm{S} 45 \mathrm{C}, 500 \mu \mathrm{m}$ bores in stainless steel and ceramics -304 and $260 \mu \mathrm{m}$ bores in zirconia material. Gears have many diverse applications in the field of automobile, rotorcraft drive system, locomotives, marine applications, railway track etc. The gears are commonly produced by conventional gear cutting methods or casting process. After manufacturing of gears, finishing of gears is a challenging task in many machining shop. It is finished by MAAF process with great accuracy [75]. The convergent and divergent nozzles made up of nitro-alloy and brass is finished by MAAF process used in hydraulic units [33]. The diesel engine fuel injector nozzles can also be finished by MAAF process. Improved quality of the nozzle characteristics is found in MAAF processed injectors resulting in enhancement of engine performance and improved emission.

\subsection{Machining of optical components}

Lenses are usually made of brittle material such as glass, which tends to fail by cracks when it is machined. Even a single micro-crack can drastically affect a lens' performance, making it completely ineffective for its intended application. Traditional methods like grinding make the process very expensive and labour intensive. To overcome these problems, MRF is developed which makes revolution in the field of optics by automate the lens finishing techniques. Highly précised lenses are used in medical equipment such as endoscopes, collision-avoidance devices for transportation industry, scientific testing devices and military's night vision equipment such as infrared binoculars. 

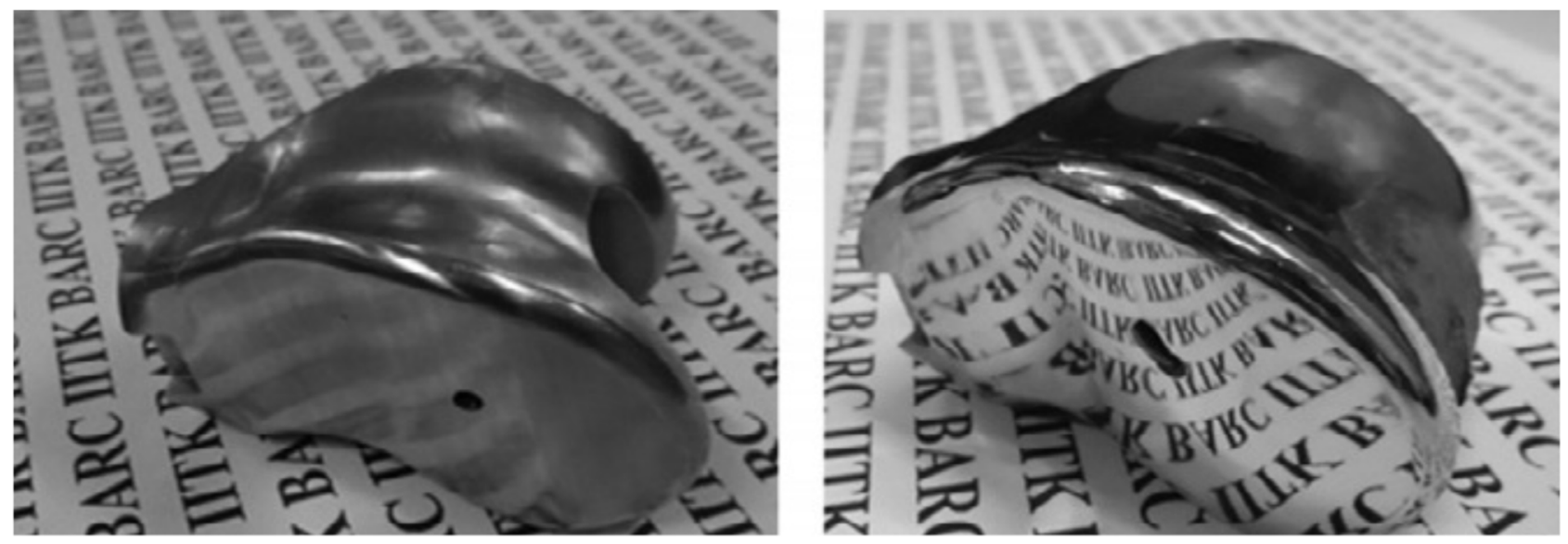

Fig. 17. Knee joint implant before and after finishing [80].

\subsection{Machining of biomedical component}

Freeform surfaces are widely used in biomedical, aerospace, turbine blades, automobile and optical components. These surfaces are commonly manufactured by rapid prototyping, casting and advanced CNC machines. After machining, finishing of these surfaces to higher level is a difficult task and many researchers have developed different types of finishing process. Sidpara et al. [66] finished knee joint implant by using magnetorheological fluid-based finishing tool and the best possible surface finish obtained through this process is $28 \mathrm{~nm}$.

Kumar et al. [80] developed R-MRAFF for finishing of freeform component similar to knee joint implant to nanometer level with uniform finishing of all faces. Figure 17 shows the knee joint implant before and after finishing through flexible brush created at the tip of polishing tool process.

\section{Research challenges of MAAF}

The MAAF is an advanced nanofinishing process but there are still some unsettled challenges exist which are explained below:

- BEMRF process is very slow process, takes large time during finishing of hard material [55];

- with MAFF [64], uniform finishing of freeform surfaces can't be achieved because of variation in the working gap between the tool and workpiece;

- in each MAAF process, owing to sedimentation problem, it is mandatory to change the MR fluid after some period of time. To avoid this problem some additives are added in MR fluid which makes these processes costly [77]. Hence, search for economical, environmental friendly and efficient process is still on;

- MRAFF process can't finish surfaces which have sharp edges because up and down motion of the MR fluid take away sharp edges first and destroy the shape of workpiece [15]. Finishing of spherical surfaces is also not possible by MAAF process;

- these MAAF processes are good for finishing the ferrous material, their rate of finishing decreases when we finish the non-ferrous materials [12]. Owing to low relative permeability of non ferrous materials as compared to ferrous materials, it is still a difficult job. Al alloy and Copper was finished through MAAF but significant finishing could not be performed due to low magnetic permeability.

\section{Research potentials}

The review presented in this article reveals an insight into a broad area of MRF and MRAFF and its process variants. However, magnificent possibilities still exist for further improvement in process efficiency of MAAF. The significant measures of MAAF process efficiency can be in terms of machining time, surface quality and economics [29]. The set-up time for MAAF depends on the design of fixtures and tooling for guiding the media. A change in workpiece geometry, in general, implies development of a new fixture. This increases the overall cost of the design and manufacture in MAAF [47]. It would be imperative to develop adjustable fixtures for different geometries so as to increase productivity of MAAF. It was revealed from the literature review that major focus was laid on glasses with simple geometry. A possible research avenue is open for finishing the materials other than optics, such as metallic, composites metallic and ceramic components used in aerospace, medical, nuclear reactor automobiles, etc., require stringent control over surface roughness. MRF and allied processes have been efficiently used for finishing of prosthesis implants, such as knee joint [64] and hip joint [49]. MRF is very efficient for finishing of nonmagnetic materials, but it is also necessary to explore the application of MRF for magnetic materials. Only a few reports are available where MRF and allied processes are used for finishing of magnetic materials [37]. Further development in this regard will provide an alternate and efficient way of Nano finishing of the components made of magnetic materials finishing of metals or hard materials by standard MR fluid is not appropriate because of low volumetric MRR, which may result in high processing time. Therefore, it is necessary to increase the aggressiveness of MR fluid by addition of suitable chemicals, which soften the metallic surface and assist the abrasive particles in mechanical abrasion. 
It is observed that the working gap does not remain constant when the MRFF tool moves over the freeform surface. As a result, the stiffness (yield stress) of the MR fluid is also different at different working gap so there is large variation in final surface roughness [25]. Thus fixture and special tooling should be designed for getting uniform results. Mostly the results available in the literature are for flat, cylindrical surfaces. Limited research literature is available on finishing of freeform surfaces and for sphere surfaces by MAAF. The use of electromagnet in these MAAF process for generating magnetic field may cause heating problem. Hence the viscosity at the end phase of polishing tool is decreased and the use of electromagnet makes the setup complicated and costly. So there is demand of the process to overcome this heating problem or the use of permanent magnet instead of electromagnet. There is also need to monitor the MAAF process online for optimization of the whole process. The experimental results should be optimized by the theoretical model.

\section{Conclusion}

This paper presented an overview of recent developments in MAAF process and their critical parameters. The major conclusions of the study are as follows:

- the MAAF is a nano-finishing technique for surface finishing. It can finish both internal and external surface with great control over forces;

- media act as a self-deformable multiple cutting tools. It changes its shape according to the complexity of the workpiece; many attempts have been made to develop efficient, environmental friendly, cost effective media;

- the BEMRF process shows better results for magnetic material as compared to non-magnetic material. This process can finish any shape of workpiece. But time taken by the process is very high;

- the permanent magnet was used in place of electromagnet coil to avoid heating problem and to finish diamagnetic copper material in less time;

- for getting better result with hard stainless steel material the motion is given to MR fluid;

- in R-MRAFF process rotational motion is provided to freeform workpiece for getting uniform roughness value of all faces;

- the MRFF was tried with three different types of MR fluid to decrease the finishing time. Chemical reactions are performed on the workpiece surface to soften it before finishing.

\section{References}

1. I. Lazoglu, Tool path optimization for free form surface machining, CIRP Ann. Manuf. Technol. 58 (2009) 101-104

2. A. Lasemi, Recent development in CNC machining of freeform surfaces: a state-of-the-art review, Comput. Aided Des. 42 (2010) 641-654

3. J.A. Schey, Introduction to manufacturing processes, Second edition, McGraw Hill, 1987
4. H. Foellinger, Optimum parameters for dressing process of conventional grinding, in: Proceedings superabrasives' 85 , 1985, pp. 8-22

5. C.J. Evans, E. Paul, D.A. Dornfeld, Lucca, G. Byrne, M. Tricard, F. Klocke, O. Dambon, B.A. Mullany, Material removal mechanisms in lapping and polishing, CIRP Ann. 52 (2003) 611-633

6. A.J. Troglio, Performance evaluation of multi-stone honing tool by experimental design methods, in: Proc. Int. Honing 2003 Conf, MR03-232, 2003, pp. 1-24

7. S. Jha, V.K. Jain, Micromanufacturing and nanotechnology. Springer, Berlin, Heidelberg, 2006, 171-195

8. M.P. Groover, Fundamentals of modern manufacturing, Prentice Hall, 1996

9. N. Taniguchi, Current status in, and future trends of ultraprecision machining and ultrafine material processing, Ann. CIRP 32/2 (1983) 573-582

10. Y. Mori, N. Ikawa, K. Sugiyama, Elastic emission machining - stress field and fracture mechanism, Technology Reports of the Osaka University, 28, 1976, pp. 525-534

11. E.G. Spencer, P.H. Schmidt, Ion beam techniques for device fabrication, J. Vac. Sci. Technol. 8 (1972) S52-S70

12. S. Singh, H.S. Shan, P. Kumar, Wear behavior of materials in magnetically assisted abrasive flow machining, J. Mater. Process. Technol. 128 (2002) 155-156

13. A. Sadiq, M.S. Shunmugam, Magnetic field analysis and roughness prediction in magnetorheological abrasive honing (MRAH), Mach. Sci. Technol. 13 (2009b) 246-268

14. J.D. Kim, Development of a magnetic abrasive jet machining system for internal polishing of circular tubes, J. Mater. Process. Technol. 71 (1997) 384-393

15. S. Jha, V.K. Jain, Design and development of magnetorheological abrasive flow finishing process, Int. J. Mach. Tool Manuf. 44 (2004) 1019-1029

16. G. Yang, B.F. Spencer Jr, J.D. Carlson, M.K. Sain, Largescale MR fluid dampers: modeling and dynamic performance considerations, Eng. Struct. 24 (2002) 309-323

17. M. Das, V.K. Jain, P.S. Ghoshdastidar, Nanofinishing of flat workpieces using rotational-magnetorheological abrasive flow finishing (R-MRAFF) process, Int. J. Adv. Manuf. Technol. 62 (2012) 405-420

18. S. Jha, V.K. Jain, R. Komanduri, Effect of extrusion pressure and number of finishing cycles on surface roughness in magnetorheological abrasive flow finishing (MRAFF) process, Int. J. Adv. Manuf. Technol. 33 (2006) 725-729

19. G.Z. Kremen, Machining time estimation for magnetic abrasive processes, Int. J. Prod. Res. 32 (1994) 2817-2825

20. V.K. Jain, P. Kumar, P.K. Behera, S.C. Jayswal, Effect of working gap and circumferential speed on the performance of magnetic abrasive finishing process, Wear 250 (2001) 384-390

21. T. Shinmura, Study on magnetic abrasive finishing characteristics of finished surface, J. Jpn. Soc. Precis. Eng. 53 (1987) 1791-1793

22. T.S. Bedi, A.K. Singh, Magnetorheological methods for nano finishing-a review, Part. Sci. Technol. 34 (2015) 412-422

23. K. Geels, D. Fowler, W-U. Kopp, M. Rückert, editors, Metallographic and materialographic specimen preparation, light microscopy, image analysis and hardness testing, ASTM International, West Conshohocken, 2007, pp. 19428-22959

24. Y. Tani, K. Kawata, Development of high-efficient fine finishing process using magnetic fluid, Ann. CIRP 33 (1984) 1 
25. S.N. Shafrir et al., Zirconia coated carbonyl iron particlebased magnetorheological for polishing, Proc. SPIE $\mathbf{7 4 2 6}$ (2009) 74260B

26. S.D. Jacobs, MRF with adjustable pH, Proc. SPIE 8169 (2011) 816902

27. W.I. Kordonski, D. Golini, Fundamentals of magnetorheological fluid utilization in high precision finishing, J. Intell. Mater. Syst. Struct. 10 (1999) 683-689

28. C.W. Macosko, Rheology: principles, measurements, and applications, VCH Publishers Inc, New York, 1994

29. P. Balasubramanian, T. Senthilvelan, Optimization of machining parameters in EDM process using cast and sintered copper electrodes, Procedia Mater. Sci. 6 (2014) $1292-1302$

30. R. Mathur, R. Khare, V. Chauhan, Magnetorheological Finishing of Optical Lenses, B. Tech. Project. Mechanical Eng. Dept., IIT-Kanpur, 2003

31. D.A. Khan, S. Jha, Selection of optimum polishing fluid composition for ball end magnetorheological finishing (BEMRF) of copper, in: Proceedings of 6th Int \&27th All India Manuf Tech, 2016, pp. 316-319

32. D.A. Khan, S. Jha, Synthesis of polishing fluid and novel approach for nanofinishing of copper using ball end magnetorheological finishing process, Mater. Manuf. Process. 33 (2017) 1150-1159

33. S.S. Kumar, S.S. Hiremath, Machining of internal features using the developed abrasive flow machine, in: Proceedings of the International Conference on Advances in Production and Industrial Engineering, NIT-Trichy, 2015, 12, pp. $298-303$

34. A. Sidpara, V.K. Jain, Experimental investigations into forces during magneto-rheological fluid based finishing process, Int. J. Mach. Tools Manuf. 51 (2011) 358-362

35. A. Sidpara, V.K. Jain, Nano-level finishing of single crystal silicon blank using magnetorheological finishing process, Tribol. Int. 47 (2012) 159-166

36. L. Yin, K. Ramesh, S. Wan, X.D. Liu, H. Huang, Y.C. Liu, Abrasive flow polishing of micro bores, Mater. Manuf. Process. 19 (2004) 187-207

37. V.K. Jain, Magnetic field assisted abrasive based micro-/ nano-finishing, J. Mater. Process. Technol. 209 (2009) 60226038

38. A. Sidpara, M. Das, V.K. Jain, Rheological characterization of magnetorheological finishing fluid, J. Mater. Manuf. Process. 24 (2009) 1467-1478

39. COM, Magnetorheological finishing, Article by center for optics manufacturing, 1998, http://www.opticam.rochester.edu

40. A. Sidpara, V.K. Jain, Analysis of forces on the freeform surface in magnetorheological fluid based finishing process, Int. J. Mach. Tools Manuf. 69 (2013) 1-10

41. D. Golini, W.I. Kordonski, P.R. Dumas, S. Hogan, Magnetorheological finishing (MRF) in commercial precision optics manufacturing, Proc. SPIE 3782 (1999) 80-91

42. W.I. Kordonski, Magnetorheological finishing, Int. J. Mod. Phys. B 10 (1996) 2837-2849

43. M. Das, V.K. Jain, P.S. Ghoshdastidar, Nanofinishing of flat workpieces using rotational-magnetorheological abrasive flow finishing (R-MRAFF) processm, Int. J. Adv. Manuf. Technol. 62 (2012) 405-420

44. S. Jha, V.K. Jain, R. Komanduri, Effect of extrusion pressure and number of finishing cycles on surface roughness in magnetorheological abrasive flow finishing (MRAFF) process, Int. J. Adv. Manuf. Technol. 33 (2006) 725-729
45. G.Z. Kremen, Machining time estimation for magnetic abrasive processes, Int. J. Prod. Res. 32 (1994) 2817-2825

46. V.K. Jain, P. Kumar, P.K. Behera, S.C. Jayswal, Effect of working gap and circumferential speed on the performance of magnetic abrasive finishing process, Wear 250 (2001) 384-390

47. L. Nagdeve, V.K. Jain, J. Ramkumar, Experimental Investigations into nano-finishing of freeform surfaces using negative replica of the knee joint, Procedia CIRP 42 (2016) 793-798

48. A.K. Singh, S. Jha, P.M. Pandey, Performance analysis of ball end magnetorheological finishing process with MR polishing fluid, Mater. Manuf. Process. 30/12 (2015) 1482-1489

49. J.K. Sutton, Orthopedic component manufacturing method and equipment, U.S. Patent No 7959490B2, 2011

50. S. Jha, V.K. Jain, Modeling and simulation of surface roughness in magnetorheological abrasive flow finishing (MRAFF) process, Wear 261 (2006) 856-866

51. V. Grover, A.K. Singh, A novel magnetorheological honing process for nano-finishing of variable cylindrical internal surfaces, Mater. Manuf. Process. 32 (2017) 573-580

52. R.K. Jain, V.K. Jain, Optimum selection of machining conditions in abrasive flow machining using neural network, J. Mater. Process. Technol. 108 (2000) 62-67

53. V. Grover, A.K. Singh, Analysis of particles in magnetorheological polishing fluid for finishing of ferromagnetic cylindrical workpiece, Part. Sci. Technol. 36 (2017) 799-807

54. S. Howard, R. Tanner, Shear rate dependence of the normal force of a magnetorheological suspension, Rheol. Acta 42 (2003) 166-170

55. N. Ida, Engineering electromagnetics, Springer-Verlag Inc., New York, 2000, pp. 625-628

56. S.K. Paswan, T.S. Bedi, A.K. Singh, Modeling and simulation of surface roughness in magnetorheological fluid based honing process, Wear 376-377, Part B (2016) $1207-1221$

57. A.K. Singh, S. Jha, P.M. Pandey, Nanofinishing of a typical 3D ferromagnetic workpiece using ball end magnetorheological finishing process, Int. J. Mach. Tools Manuf. 63 (2012) 21-31

58. A.C. Wang, L. Tsai, C.H. Liu, K.Z. Liang, S.J. Lee, Elucidating the optimal parameters in magnetic finishing with gel abrasive, Mater. Manuf. Process. 26 (2011) 786-791

59. M. Barletta, S. Guarino, G. Rubino, V. Tagliaferri, Progress in fluidized bed assisted abrasive jet machining (FB-AJM) internal polishing of aluminum tubes, Int. J. Mach. Tools Manuf. 47 (2007) 483-495

60. S.O. Kim, J.S. Kwak, Magnetic force improvement and parameter optimization for magnetic abrasive polishing of AZ31 magnesium alloy, Trans. Nonferrous Met. Soc. China 18 (2008) 369-373

61. V. Grover, A.K. Singh, A novel magnetorheological honing process for nano-finishing of variable cylindrical internal surfaces, Mater. Manuf. Process. 32 (2017) 573-580

62. P. Balasubramanian, T. Senthilvelan, Optimization of machining parameters in EDM process using cast and sintered copper electrodes, Procedia Mater. Sci. 6 (2014) 1292-1302

63. A.K. Singh, S. Jha, P.M. Pandey, Design and development of nanofinishing process for 3D surfaces using ball-end MR finishing tool, Int. J. Mach. Tools Manuf. 51 (2011) 142-151 
64. A. Sidpara, V.K. Jain, Nanofinishing of freeform surfaces of prosthetic knee joint implant, Proc. Inst. Mech. Eng. B: J. Eng. Manuf. 226 (2012) 1833-1846

65. V.K. Jain, P. Ranjan, V.K. Suri, R. Komanduri, Chemomechanical magneto-rheological finishing (CMMRF) of silicon for microelectronics applications, CIRP Ann. Manuf. Technol. 59 (2010) 323-328

66. P.M. Pandey, R.S. Mulik, Ultrasonic assisted magnetic abrasive polishing of hardened AISI 52100 steel using unbounded Sic abrasives. Int. J. Refract. Met. H 29 (2011) 68-77

67. B. Anwesa, B. Das, Design and fabrication of a novel polishing tool for finishing freeform surfaces in magnetic field assisted finishing (MFAF) process, Precis. Eng. 49 (2017) 61-68

68. H. Kumar, A.K. Singh, G. Vishwas, Magnetorheological nano-finishing of diamagnetic material using permanent magnets tool, Precis. Eng. 51 (2018) 30-39

69. Y. Hayashi, T. Nakajima, T. Kunio, Ultra uniform Chemical Mechanical Polishing (CMP) using a "Hydro Chuck", featured by wafer mounting on a quartz glass plate with fully flat water supported surface, Jpn. J. Appl. Phys. 35 (1996) 1054-1059

70. P. Ranjan, R. Balasubramaniam, V.K. Suri, Development of chemo-mechanical magnetorheological finishing process for super finishing of copper alloy, Int. J. Manuf. Technol. Manag. 27 (2013) 130-141

71. V.C. Shukla, P.M. Pandey, Comprehensive study for optimizing sintered density of magnetic abrasive particles of $\mathrm{Fe}$ and $\mathrm{SiC}$ mix for UAMAF process, IVth International Conference on Production and Industrial Engineering, CPIE, NIT Jalandhar, India, 2016

72. T. Mori, K. Hirota, Y. Kawashima, Clarification of magnetic abrasive finishing mechanism, J. Mater. Process. Technol. 143-144 (2003) 682-686

73. V.C. Shukla, P.M. Pandey, Experimental investigations into sintering of magnetic abrasive powder for ultrasonic assisted magnetic abrasive finishing process, Mater. Manuf. Process. 32 (2017) 108-114

74. R.S. Mulik, P.M. Pandey, Mechanism of surface finishing in ultrasonic-assisted magnetic abrasive finishing process, Mater. Manuf. Process. 25 (2010) 1418-1427

75. H. Yamaguchi, T. Shinmura, Study of the surface modification from an internal magnetic abrasive finishing process, Wear 225-229 (1999) 246-255
76. P. Kala, P.M. Pandey, Experimental study on finishing forces in double disk magnetic abrasive finishing process while finishing paramagnetic workpiece, Procedia Mater. Sci. 5 (2014) 1677-1684

77. D.J. Klingenberg, Magneto rheology: applications and challenges, AIChE J. 47 (2001) 246-249

78. M.S. Niranjan, S. Jha, Performance analysis of ball end magnetorheological Finishing using sintered magnetic abrasive based magnetorheological polishing fluid, 5th International \& 26th All India Manufacturing Technology, Design and Research Conference (AIMTDR 2014), IIT Guwahati, Assam, India, 2014

79. A. Sadiq, M.S. Shunmugam, Investigation into magnetorheological abrasive honing (MRAH), Int. J. Mach. Tools Manuf. 49 (2009) 554-560

80. S. Kumar, V.K. Jain, A. Sidpara, Nanofinishing of freeform surfaces (knee joint implant) by rotational-magnetorheological abrasive flow finishing (R-MRAFF) process, Precis. Eng. 42 (2015) 165-178

81. J.M. Ginder, in: G.L. Trigg (Ed.), Encyclopedia of applied physics Vol. 16, Wiley, New York, 1996, p. 487

82. W.I. Kordonski, S. Gorodkin, R. Behlok, In-line monitoring of (MR) fluid properties, J. Magn. Magn. Mater. 2015 (2015) 328-334

83. H. Yarmand, S. Gharehkhani, Numerical investigation of heat transfer enhancement in a rectangular heated pipe for turbulent nanofluid, Hindawi Publishing Corporation Scientific World Journal, Vol. 2014, (2014) Article ID 369593

84. A. Sidpara, V.K. Jain, Experimental investigations into forces during magneto-rheological fluid based finishing process, Int. J. Mach. Tools Manuf. 51 (2011) 358-362

85. M. Das, V.K. Jain, P.S. Ghoshdastidar, Fluid flow analysis of magnetorheological abrasive flow finishing (MRAFF) process, Int. J. Mach. Tools Manuf. 48 (2008) 415-426

86. A.K. Singh, S. Jha, P.M. Pandey, Mechanism of material removal in ball end magnetorheological finishing process, Wear 302 (2013) 1180-1191

87. A. Zafar, S. Jha, Reprint of modeling of surface roughness in ball end magnetorheological finishing (BEMRF) process, Wear 376-377 (2017) 194-202

Cite this article as: Chinu Kumari, Sanjay Kumar Chak, A review on magnetically assisted abrasive finishing and their critical process parameters, Manufacturing Rev. 5, 13 (2018) 